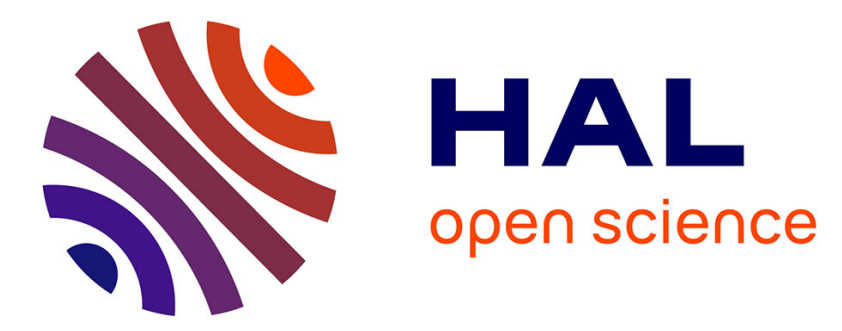

\title{
Design of a PI Control using Operator Theory for Infinite Dimensional Hyperbolic Systems
}

Valérie dos Santos, Yongxin Wu, Mickael Rodrigues

\section{To cite this version:}

Valérie dos Santos, Yongxin Wu, Mickael Rodrigues. Design of a PI Control using Operator Theory for Infinite Dimensional Hyperbolic Systems. IEEE Transactions on Control Systems Technology, 2014, 22 (5), pp.2024 - 2030. 10.1109/TCST.2014.2299407 . hal-00936398

\section{HAL Id: hal-00936398 https://hal.science/hal-00936398}

Submitted on 25 Jan 2014

HAL is a multi-disciplinary open access archive for the deposit and dissemination of scientific research documents, whether they are published or not. The documents may come from teaching and research institutions in France or abroad, or from public or private research centers.
L'archive ouverte pluridisciplinaire HAL, est destinée au dépôt et à la diffusion de documents scientifiques de niveau recherche, publiés ou non, émanant des établissements d'enseignement et de recherche français ou étrangers, des laboratoires publics ou privés. 


\title{
Design of a PI Control using Operator Theory for Infinite Dimensional Hyperbolic Systems
}

\author{
Valérie Dos Santos Martins, Yongxin Wu, Mickael Rodrigues
}

\begin{abstract}
This paper considers the control design of a nonlinear distributed parameter system in infinite dimension, described by the hyperbolic Partial Differential Equations (PDEs) of de Saint-Venant. The nonlinear system dynamic is formulated by a Multi-Models approach over a wide operating range, where each local model is defined around a set of operating regimes. A new Proportional Integral (PI) feedback is designed and performed through Bilinear Operator Inequality (BOI) and Linear Operator Inequality (LOI) techniques for infinite dimensional systems. The new results have been simulated and also compared to previous results in finite and infinite dimension, in order to illustrate the new theoretical contribution.
\end{abstract}

\section{Index Terms}

PDEs, de Saint-Venant Equations, Multi-Models, Semigroup Theory, IMBC.

\section{INTRODUCTION}

Regulation of irrigation channels has received an increasing interest over the last three decades. Water losses in open channels are very large due to inefficient management and control. To avoid overflows and satisfy the water request, the level of instrumentation (e.g., operating motor-driven gates, water level measurements) and automation in open channel networks increase [24]. In order to deliver water, it is important to ensure that the water level and the flow rate in open channels remain at certain values. The difficulty of this regulation problem is that only the gates positions can meet performance specifications. Such problems can be solved by designing boundary control laws in order to satisfy the control objectives: to maintain water level or flow rate at given values.

M. Wu, M. Rodrigues and Ms. Dos Santos Martins are with LAGEP, Université de Lyon, Lyon, F-69003, France; Université Lyon 1, CNRS, UMR 5007, LAGEP, Villeurbanne, F-69622, France. e-mail: name@lagep.univ-lyon1.fr. 
The open surface channels couple transport phenomena and delay phenomena, so they have a complex nonlinear dynamics. In this case, the distributed parameter systems have a dynamic represented by hyperbolic Partial Differential Equations (PDE): the equations of de Saint-Venant, which depend on time and space [23], [27], [35]. Some studies take into account the uncertainties and apply a robust control approach [22], [21]. Studying directly the nonlinear dynamics is also possible as in [10], [14], [21], [36]. The Riemann approach has also been used to prove stability results for systems of two conservation laws [18], and for systems of larger dimensions in [20]. Recently, it has been also coupled with LMI [2]. The Lyapunov techniques have been used in [5], [9], [10].

In practice, industrial processes such as mining, chemical or water treatment processes are characterized by complex systems which operate in multiple operating regimes. Multi-Models methods split the operating range of a system into separate regions where local models are affected to each region [26] for control and Fault Diagnosis purposes [3], [16], [28]. Each local model is defined as a Linear Time Invariant (LTI) model for each operating point. The MultiModels philosophy is based on weighting functions which ensure the transition between the different local models. Some authors speak about gain scheduling strategy for example in [19] or Linear Parameter Varying (LPV) controllers [30].

The use of Multi-Models representation for the study of the stability of a system described by nonlinear PDE has been examined in [2], [12], [13]. The nonlinear PDE stability is studied by transferring the common approaches based on finite dimension to infinite dimension. The theoretical proof has been given for the closed loop stability under a Proportional and a Proportional Integral (PI) controller with identical gains. In this paper, an analysis of the closed loop stability of the de Saint-Venant PDE is proposed with a general PI, using Multi-Models and the Internal Model Boundary Control (IMBC) structure. A variable elimination technique, as for finite-dimensional systems [4], [29], [32], has been used in order to solve a BOI (well known as Bilinear Matrix Inequality (BMI) in finite dimension) problem by the resolution of two LOI (well known as Linear Matrix Inequalities (LMI) in finite dimension).

The paper is organized as follows: firstly, the equations of de Saint-Venant are presented as well as the control problem. The Internal Model Boundary Control is explained and the physical constraints are given. In section II, the linearized models are defined around equilibrium sets as their insertion into the LOI formalism. Part III of the paper is dedicated to the design of 
the feedback gains by LOI \& BOI techniques which ensures the stability of the system. A Proportional Integral (PI) controller is implemented based on two propositions: these are the major contributions of this paper. The last section IV is dedicated to simulations. Comparisons between the simulations with the PI controller (for which the proportional gain is equal to the integral gain, developed in previous works) and the new PI controller $\left(K_{\text {int }} \neq K_{p r}\right.$ calculated using the LOI \& BOI techniques) are realized. Simulations of a new channel are also implemented in the last section.

\section{Problem StATEMENT ABout CHANNEL REgulation}

The control problem concerns the stabilization of the water flow rate and/or the water height around an equilibrium for a reach denoted by $\left(Z_{e}(x), Q_{e}(x)\right)$.

\section{A. A model of a reach}

The channel is supposed to have a sufficient length $L$ (from $x=0$ to $x=L$ ) such that one can consider that the lateral movement is uniform. $Q(x, t)$, the water flow rate, and $Z(x, t)$, the height of the water, are the state variables. The nonlinear PDE of de Saint-Venant which describe the flow on the channel are [8], [17]:

$$
\begin{aligned}
& \left\{\begin{array}{l}
\partial_{t} Z=-\partial_{x} \frac{Q}{b}, \\
\partial_{t} Q=-\partial_{x}\left(\frac{Q^{2}}{b Z}+\frac{1}{2} g b Z^{2}\right)+g b Z(I-J),
\end{array}\right. \\
& y(t)=C[Z(x, t) \quad Q(x, t)]^{T} \\
& Z_{0}(x)=Z(x, 0), Q_{0}(x)=Q(x, 0)
\end{aligned}
$$

$\forall x \in \Omega=\left(x_{u p}, x_{d o}\right)=(0, L), t>0, C:\left(L^{2}(0, L)\right)^{2} \rightarrow \mathbb{R} . I$ is the slope, $b$ is the channel width, $g$ is the gravity constant. $J$ is the friction slope from the formula of Manning-Strickler and $R$ is the hydraulic radius. The considered boundary conditions $\forall x \in \Gamma=\partial \Omega$ are two underflow gates. The controlled variable is defined as follows:

$$
Q(x, t)=U(t) \Psi(Z(x, t))
$$

with $\Psi(Z)=\kappa \sqrt{2 g\left(Z_{u p}-Z_{d o}\right)}$. $Z_{u p}$ is the water height at upstream of the gate, $Z_{d o}$ is the water height at downstream of the gate, $\kappa$ is the product of the channel width with the water flow rate coefficient of the gate. The gates opening $U(t)$ is the control at upstream $\left(U_{u p}=U_{0}\right)$ and at downstream $\left(U_{d o}=U_{L}\right)$. The output variable is the water level at downstream, i.e. $Z(L)$. 


\section{B. A regulation model}

The fluvial case, i.e. the subcritical case [23], is considered. Let $\xi(t)=(z(t) q(t))^{T}$ be the linearized state variable, then the model around the equilibrium state $\left(Z_{e}(x) Q_{e}(x)\right)^{T}$ is:

$$
\begin{aligned}
\partial_{t} \xi(x, t) & =\mathcal{A} \xi(x, t)=A_{1}(x) \partial_{x} \xi(x, t)+A_{2}(x) \xi(x, t) \\
F_{b} \xi(t) & =B_{b} u(t) \text { and } \xi(0, t)=\xi_{0}(t)
\end{aligned}
$$

where $A_{1}$ and $A_{2}$ are matrices of the space variable $x$. The linearized boundary conditions (6) are equivalent to:

$$
\begin{array}{r}
q\left(x_{u p}, t\right)=U_{u p, e} \partial_{z} \Psi\left(Z_{e}\left(x_{u p}, t\right)\right) z\left(x_{u p}, t\right)+u_{u p}(t) \Psi\left(Z_{e}\left(x_{u p}, t\right)\right), \\
q\left(x_{d o}, t\right)=U_{d o, e} \partial_{z} \Psi\left(Z_{e}\left(x_{d o}, t\right)\right) z\left(x_{d o}, t\right)+u_{d o}(t) \Psi\left(Z_{e}\left(x_{d o}, t\right)\right),
\end{array}
$$

where $U_{u p, e}$ and $U_{d o, e}$ are the opening gates for the upstream and downstream (respectively) at the equilibrium and $u_{u p}(t), u_{d o}(t)$ are the variations of these opening gates to be controlled. The control problem is to find the variations of $u_{u p}(t)$ at extremity $x=x_{u p}$ and $u_{d o}(t)$ at extremity $x=x_{d o}$ of the reach such that the downstream water level, $Z\left(x_{d o}, t\right)=Z(L, t)$ (measured variable) tracks a reference signal $r(t)$. The reference signal $r(t)$ is chosen for all cases either constant or non-persistent (a stable step answer of a non-oscillatory system).

In this paper, the control scheme based on the Internal Model Boundary Control (IMBC) is adopted [11]. This control strategy integrates the process model in real time and allows to regulate the water height in all the points of the channel by taking into account the error between the linearized model and the real system (or the nonlinear model for the simulations).

\section{Open-loop system stability}

Equation (5) describes the open loop system dynamics. In this representation, the state vector $\xi(x, t)$ is not explicitly linked with the boundary control. In order to design an output feedback and to study the closed-loop stability, a distribution operator $D$ of control at the boundary is introduced $[15]^{1}, D: \mathcal{C}^{k}\left([0, \infty], \mathbb{R}^{n}\right) \rightarrow\left(L^{2}(0, L)\right)^{2}$. It is a bounded operator such that $\operatorname{Im}(D)=$ $\operatorname{Ker}(\mathcal{A})$ and $D u \in D(\mathcal{A})$ and [11], [15], [33]:

$$
\xi(x, t)=\varphi(x, t)+D u(t) .
$$

\footnotetext{
${ }^{1}$ Regularity coefficient is generally taken as $k=2$
} 
This operator is naturally null in the domain of $\mathcal{A}(x)$ as it is active only on the boundary of the domain. This change of variables allows to get a Kalman representation of the system [1], [15], [33]:

$$
\begin{aligned}
\partial_{t} \varphi(x, t) & =\mathcal{A}(x) \varphi(x, t)-D \dot{u}, \\
\varphi(x, 0) & =\varphi_{0}(x)=\xi_{0}(x)-D u(0), \\
y(t) & =C \varphi(x, t)+C D u(t) .
\end{aligned}
$$

It has been proved that the open loop system (10-12) is exponentially stable [11], as the operator of the linearized system in infinite dimension generates an exponentially stable $C_{0}$-semigroup. Moreover, under a PI-control $u(t)=\alpha_{i} K_{i} \int \varepsilon(s) d s+\alpha_{p} K_{p} \varepsilon(t) \in U=\mathbb{R}^{n}, u \in C^{k}([0, \infty], U)$, the stability of the closed-loop nonlinear system is ensured under some specific conditions on the gain synthesis. They are deduced from the properties of the IMBC structure and from the stability of the closed-loop linearized system.

For example, for the tuned gains of the PI-control the stability conditions are ensured if:

$$
\begin{array}{r}
0 \leqslant \alpha_{i}<\alpha_{i, \max }=\min _{\lambda \in \Gamma}\left(a\left\|R\left(\lambda ; \mathcal{A}_{e}\right)\right\|+1\right)^{-1}, \\
0 \leqslant \alpha_{p}<\alpha_{p, \max }=\left(\sup _{\lambda \in \Gamma} a\|R(\lambda ; \mathcal{A})\|\right)^{-1},
\end{array}
$$

where $\mathcal{A}_{e}$ is a part of the series development of the closed loop operator [11], $R(\lambda ; K)$ is the resolvent operator of $K$, and $a$ is a constant which depends on $\mathcal{A}_{e}$.

These theoretical results have been corroborated by simulations and experimentations [11]. Those experimentations have shown the limitations due to the linearization around an equilibrium state. A first attempt with a Multi-Models approach has been realized with success. However, it was not optimal and no theoretical proof has been given. The first approach by an integral control [13] had been extended to a PI control in [12], but the proportional and the integral gains were equal. The aim of this paper is to extend the previous results in infinite dimension with the proportional gain different from the integral gain. The theoretical proof realized in finite dimension with $K_{\text {int }} \neq K_{p r}$ [29] is developed to infinite dimensional systems. In order to control the water level over a wide operating range, a set of models is considered around judicious operating regimes: a control is synthesized and activated on the intervals when the system gets through the intervals. 


\section{A Multi-Models representation of de Saint-Venant's Equation}

The Multi-Models representation [29], [13] of de Saint-Venant's PDE around $N$ operating points is defined by the following equations:

$$
\begin{aligned}
\partial_{t} \xi(x, t) & =\sum_{i=1}^{N} \mu_{i}(\zeta(t)) \mathcal{A}_{i}(x) \xi(x, t) \text { with } \mathcal{A}_{i}(x)=A_{1, i}(x) \partial_{x}+A_{2, i}(x), \\
\xi_{0}(x) & =\xi(x, 0),
\end{aligned}
$$

where $\mathcal{A}_{i}(x)$ is the operator which corresponds to the $i^{t h}$ equilibrium state. $\zeta(t)$ is a function which depends on some decision variables directly linked with the measurable state variables and eventually to the input. $\mu_{i}(\zeta(t))$ are the weighting functions which activate the control law in function of the output of the process $Z_{L}$. They belong to a convex set such that

$$
\sum_{i}^{N} \mu_{i}(\zeta(t))=1 \text { and } \mu_{i}(\zeta(t)) \geq 0 .
$$

In the following section, the control law synthesis by LOI techniques is considered.

\section{STUDY OF THE CLOSED-LOOP SYSTEM STABILITY BY LOI}

In this part, the closed loop structure is studied under a proportional integral feedback. Recall that the aim is to control the water height over all the operating range, so the output $y(t)$ is not the variations around an equilibrium but the total water height. To this end, the output $y(t)$ is modified:

$$
y(t)=C \xi(x, t)+E q(x, t)
$$

where $E q(x, t)=\sum_{i=1}^{N} \mu_{i}(\zeta(t))\left(Z_{e, i}(x, t) Q_{e, i}\right)^{T}$ is the equilibrium state and for this paper $C E q(x, t)=\sum_{i=1}^{N} \mu_{i}(\zeta(t)) Z_{e, i}(L)$ as the aim is to regulate the water level at $x=L$.

\section{A. Closed-loop structure for a proportional integral feedback}

Let $K_{\text {int }}$ and $K_{p r}$ be the integral and proportional gains respectively. It follows that [11]:

$$
u(t)=K_{\text {int }} \int[r(\tau)-y(\tau)] d \tau+K_{p r}[r(t)-y(t)]
$$

where $r(t)$ is the physical water level wanted (not the variations). So by using (9) one gets:

$$
y(t)=C \varphi(x, t)+C E q(x, t)+C D u(t),
$$


and by replacing $y(t)$ into the equation (16), it becomes:

$$
\begin{aligned}
u(t)= & K_{\text {int }} \int[r(\tau)-(C \varphi(x, \tau)+C E q(x, \tau)+C D u(\tau))] d \tau \\
& +K_{p r}[r(t)-(C \varphi(x, t)+C E q(x, t)+C D u(t))]
\end{aligned}
$$

In each local model, $E q(x, t)$ is a piecewise function $(\dot{E} q(x, t)=0)^{2}$. This is also the case of $r(t)$. So, $\dot{u}$ can be simplified to:

$$
\dot{u}(t)=K_{\text {int }}[r(t)-C \varphi(x, t)-C E q(x, t)-C D u(t)]-K_{p r} C \mathcal{A}_{i}(x) \varphi(x, t)
$$

By replacing $\dot{u}$ into the equation (10) and with $\tilde{K}_{\text {int }}=D K_{i n t}, \tilde{K}_{p r}=D K_{p r}$, the expression of the closed-loop system can be expressed as follows:

$$
\begin{aligned}
\partial_{t} \varphi(x, t)=\sum_{i=1}^{N} & \mu_{i}(\zeta(t))\left[\left(\mathcal{A}_{i}(x)+\tilde{K}_{\text {int }} C+\tilde{K}_{p r} C \mathcal{A}_{i}(x)\right) \varphi(x, t)\right. \\
& \left.\quad+\tilde{K}_{\text {int }}(C D u(t)+C E q(x, t)-r(t))\right]=\sum_{i=1}^{N} \mathcal{M}_{i}(x, t) .
\end{aligned}
$$

The stability conditions are ensured by using a quadratic Lyapunov function [30] in order to guarantee the convergence of the water height to the reference $r(t)$ over the widest operating range.

\section{B. Stability study with a Lyapunov function}

Let us consider:

$$
V(\varphi(x, t), t)=\langle\varphi(x, t), P \varphi(x, t)\rangle
$$

where $\langle.,$.$\rangle is the considered inner product. The Multi-Models representation of the linearized$ PDE of de Saint-Venant defined by equation (20) is asymptotically stable if there exists an operator $P>0$, such that:

$$
\langle\dot{\varphi}, P \varphi\rangle+\langle\varphi, P \dot{\varphi}\rangle=-\langle\varphi, \varphi\rangle
$$

The main difference here between the stability result in finite and infinite dimension [13], lies in the inequality of the Lyapunov function for finite dimensional systems and equality for infinite ones (22). This equality complexity can be removed in some cases; as for example for operators

\footnotetext{
${ }^{2}$ The following notation is considered: $\partial_{t} \phi=\dot{\phi}$ whatever the function $\phi$.
} 
with compact resolvent [6], [11], [34] or [31]. In this case, the same inequality from finite dimension is a sufficient and necessary condition for the infinite dimensional case; it needs to satisfy the spectral growth assumption [34], [15]. Moreover, for the equations of de Saint-Venant, it has been shown that the operator has a compact resolvent [11] so it satisfies the spectral growth assumption. Then, by taking into account (20)-(22), one has to prove the following inequality:

$$
\left\langle\mathcal{M}_{i}, P \varphi\right\rangle+\left\langle\varphi, P \mathcal{M}_{i}\right\rangle<0
$$

where $\mathcal{M}_{i}$ is defined in (20).

The development of the inequality (23) leads us to consider an inequality for each local system of index i such that ${ }^{3}$ :

$$
\begin{aligned}
& \left\langle\left[\mathcal{A}_{i}+\tilde{K}_{i n t} C+\tilde{K}_{p r} C \mathcal{A}_{i}\right] \varphi(., t), P \varphi(., t)\right\rangle+\left\langle\tilde{K}_{\text {int }}[C D u(t)+C E q(., t)-r(t)], P \varphi(., t)\right\rangle \\
& +\left\langle\varphi(., t), P\left[\mathcal{A}_{i}+\tilde{K}_{i n t} C+\tilde{K}_{p r} C \mathcal{A}_{i}\right] \varphi(., t)\right\rangle+\left\langle\varphi(., t), P \tilde{K}_{i n t}[C D u(t)+C E q(., t)-r(t)]\right\rangle \quad<0 .
\end{aligned}
$$

In the inequality (24), which defines the stability condition of the system $\forall i$, the control parameter $u$ appears; this is a difficulty for the gain synthesis: $\tilde{K}_{i n t}, \tilde{K}_{p r}$.

A first approach was made in [12] with $K_{p r}=K_{\text {int }}$. In this paper, the previous results are improved as $K_{\text {int }}$ is considered different from $K_{p r}$. It has been proved that a good choice of $K_{\text {int }}$ and $K_{p r}$ based on semigroup theory is $K_{\text {int }}=-\alpha_{i}[C D]^{\dagger}$ and $K_{p r}=\alpha_{p}[C D]^{\dagger}$ (where $\dagger$ stands for the right pseudo-inverse) in [11]. $\alpha_{i}$ and $\alpha_{p}$ are defined in (13)-(14). So, one can assume that $\exists \beta \in \mathbb{R}$ such that $K_{p r}=\beta K_{\text {int }}$, i.e. $\tilde{K}_{p r}=\beta \tilde{K}_{\text {int }}$. Then, the equation (24) becomes:

$\left\langle\left[\mathcal{A}_{i}+\tilde{K}_{\text {int }} C+\beta \tilde{K}_{\text {int }} C \mathcal{A}_{i}\right] \varphi(., t), P \varphi(., t)\right\rangle+\left\langle\tilde{K}_{\text {int }}[C D u(t)+C E q(., t)-r(t)], P \varphi(., t)\right\rangle$

$+\left\langle\varphi(., t), P\left[\mathcal{A}_{i}+\tilde{K}_{\text {int }} C+\beta \tilde{K}_{\text {int }} C \mathcal{A}_{i}\right] \varphi(., t)\right\rangle+\left\langle\varphi(., t), P \tilde{K}_{\text {int }}[C D u(t)+C E q(., t)-r(t)]\right\rangle<0$

Note that the open-loop system (5)-(8) is exponentially stable as the closed loop one under a PI-control, with gains correctly tuned [11] for a time $t$ well chosen. So, one can assume that $\exists k>0$, such that:

$$
|C \xi(x, t)+C E q(x, t)-r(t)| \leq k|C \varphi(x, t)|
$$

and with $\alpha=(k+1) \varepsilon_{\left(\varphi^{T} P \tilde{K}_{i n t} C \varphi\right)}[13]$ :

$$
\left\langle\varphi, P \tilde{K}_{i n t}(C D u(x, t)+C E q(x, t)-r(t))\right\rangle \leq\left\langle\varphi, \alpha P \tilde{K}_{i n t} C \varphi\right\rangle
$$

${ }^{3}$ Due to the lack of space, $(x, t)$ is replaced by $(., t)$. 
For finite dimensional systems, a stability study has been given in our paper [29], by using well-known linear techniques, but not developed for infinite dimensional systems. Here, the main contribution consists in a tool developed using the semigroup theory.

Proposition 1: Let $Z$ be a Hilbert space and let $G, U, V, X$ four linear operators on $Z$ such that $G: D(G) \subset Z \rightarrow D(G)$. The domains of $U, V$ and $X$ are densely defined on $D(G)$, the domain of $G$. $X$ is a self-adjoint operator such that $\|X\| \leq 2 \sigma$.

If $\exists \sigma \in \mathbb{R} \backslash\{0\}$ which satisfy, $\forall \phi, \varphi \in D(G)$

$$
\begin{aligned}
& \left\langle G \varphi+U^{*} \phi, \varphi\right\rangle+\left\langle U \varphi+\sigma^{-1} \phi, \phi\right\rangle<0 \\
& \left\langle G \varphi+V^{*} \phi, \varphi\right\rangle+\left\langle V \varphi+\sigma^{-1} \phi, \phi\right\rangle<0
\end{aligned}
$$

then, the following inequality is also satisfied:

$$
\langle G \varphi, \varphi\rangle+\left\langle U^{*} X V \varphi, \varphi\right\rangle+\left\langle V^{*} X U \varphi, \varphi\right\rangle<0
$$

Proof: See appendix.

The following proposition extends our results [29] to infinite dimensional systems.

Proposition 2: If there exists a self-adjoint operator $P$, matrices $W_{\text {int }}$ and $W_{p r}$, scalars $\sigma, \gamma \in$ $\mathbb{R}$, such that the inequalities (28) are satisfied with $G=\mathcal{A}_{i}^{*} P+P \mathcal{A}_{i}+\gamma W_{\text {int }} C+\gamma C^{*} W_{\text {int }}^{*}$, $U^{*}=W_{\text {int }}, V=C \mathcal{A}_{i}$, then one gets the following inequalities:

$$
\begin{aligned}
& \left\langle\left(\mathcal{A}_{i}^{*} P+P \mathcal{A}_{i}+\gamma W_{i n t} C+\gamma C^{*} W_{i n t}^{*}\right) \varphi+W_{i n t} \phi, \varphi\right\rangle+\left\langle W_{i n t}^{*} \varphi+\sigma^{-1} \phi, \phi\right\rangle<0 \\
& \left\langle\left(\mathcal{A}_{i}^{*} P+P \mathcal{A}_{i}+\gamma W_{i n t} C+\gamma C^{*} W_{i n t}^{*}\right) \varphi+\left(C \mathcal{A}_{i}\right)^{*} \phi, \varphi\right\rangle+\left\langle C \mathcal{A}_{i} \varphi+\sigma^{-1} \phi, \phi\right\rangle<0
\end{aligned}
$$

and the closed-loop system (20) under the PI control law (16) is stable.

Proof: Let us consider (21) with the closed-loop system (20) under a PI control law (16). By considering the inequality (23), we can obtain (25) with $K_{p r}=\beta K_{\text {int }}$. Now, let us assume that the inequality (27) holds true, then (25) becomes:

$$
\left\langle\left(P\left[\mathcal{A}_{i}+\gamma \tilde{K}_{i n t} C+\beta \tilde{K}_{i n t} C \mathcal{A}_{i}\right]\right) \varphi, \varphi\right\rangle+\left\langle\varphi,\left(P\left[\mathcal{A}_{i}+\gamma \tilde{K}_{i n t} C+\beta \tilde{K}_{i n t} C \mathcal{A}_{i}\right]\right) \varphi\right\rangle<0
$$

with $\gamma=1+\alpha, \tilde{K}_{i n t}=P^{-1} W_{i n t}, \tilde{K}_{p r}=\beta \tilde{K}_{i n t}$. The inequality (42) has two variables: $\beta$ and $W_{\text {int }}$ that lead to a BOI problem. By using proposition 1, the BOI problem can be solved as two LOI problem. The inequality (42) is equivalent to (40) with $G=\mathcal{A}_{i}^{*} P+P \mathcal{A}_{i}+\gamma W_{i n t} C+\gamma C^{*} W_{\text {int }}^{*}$, $U^{*}=W_{\text {int }}, V=C \mathcal{A}_{i}, X=\beta I d$. Proposition 1 allows to conclude that if the inequalities (41) 
are satisfied, the Lyapunov inequality is also true. Thus the system (15) under PI control is stable.

Remark 3: The inequalities (41) seem to be linked with the stability of each submodel in infinite dimension, with a Lyapunov Input-to-state stability (ISS) function [25], [7].

The aim of section IV is to compare the simulated curves obtained with this method and the ones

obtained on the experimental benchmark [11] and the simulations with the gains $\tilde{K}_{i n t}=\tilde{K}_{p r}$ [12], [13].

\section{Simulation Results}

Two benchmarks are used for the simulations: the micro-channel of Valence (France) and the channel of Gignac (France). The simulations are based on a Chang and Cooper scheme, for more details see [10], [13].

For both applications, the weighting function $\mu_{i}(\zeta(t))$ is equal to 1 if the output's height is included into the validity domain of the model and 0 in the other case for each operating state. The output of the system is the decision variable. The parameter $\zeta(t)$ is a function of it. Both coefficients $\beta$ and $\sigma$ of the proposition 1 are negative in both cases simulated, so the condition $\|X\| \leq-2 \sigma$ is always satisfied whatever $X=\beta I d$. Here, the inequalities (41) are solved after discretization by LMI as in [29].

\section{A. The micro-channel of Valence}

The equilibrium profiles have been chosen such that the calculated control law from the local models can be efficient over all the operating range of the water height [11]. Notice that it has been experimentally verified that a local model is valid around $\pm 20 \%$ of an equilibrium profile. In order to assign references which are included between $0.06 \mathrm{~m}$ and $0.2 \mathrm{~m}$, the operating points at $x=0$ are given in the Table $\mathrm{I}$.

The following simulation (Figure (1.a)) compares PI controllers: one with gains $K_{\text {int }}=K_{p r}$ [12] and the new one with gains $K_{\text {int }} \neq K_{p r}$. The Figure (1.b) represents the dynamic evolution of the gates opening of the simulated system.

It will be observed in Figure (1.a) that the water height convergence with the new PI controller is better than the one obtained with $K_{\text {int }}=K_{p r}$ [12] and the overshoot is less too. It is also 


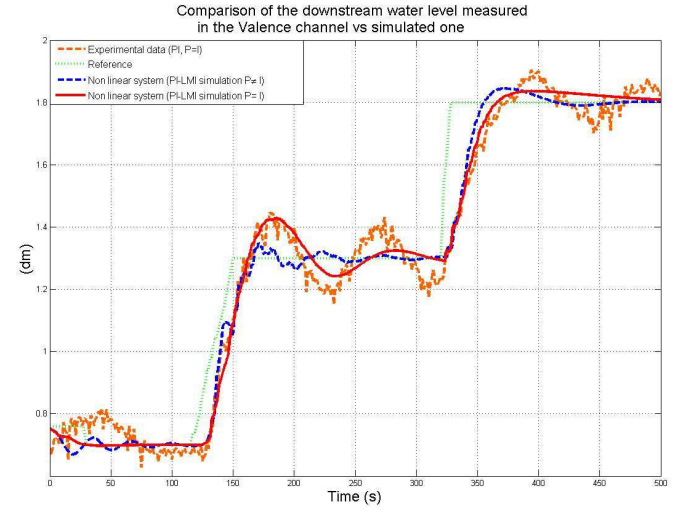

a) Comparison of the downstream water level

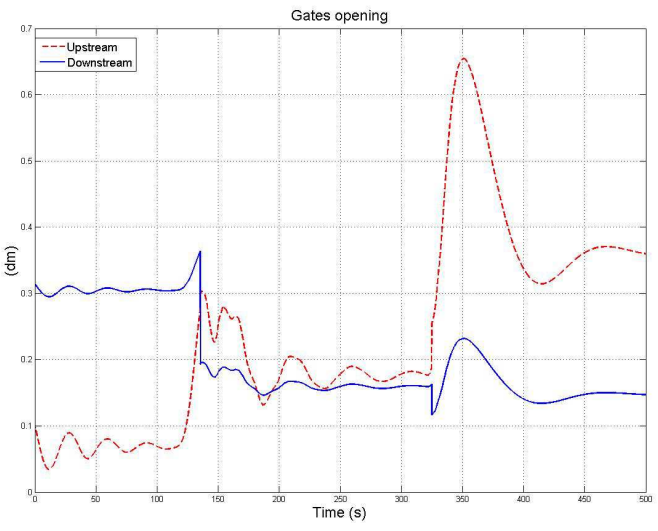

b) Gates opening

Fig. 1. Valence channel simulation

better than the experimental PI which has been implemented in [11].

TABLE I

INITIAL SET POINTS FOR THE SIMULATION OF THE CHANNELS OF VALENCE AND GIGNAC

\begin{tabular}{|l|c|c|c|}
\hline & $z_{e 1}(x=0)$ & $z_{e 2}(x=0)$ & $z_{e 3}(x=0)$ \\
\hline Valence & $0.06 \mathrm{~m}$ & $0.1 \mathrm{~m}$ & $0.16 \mathrm{~m}$ \\
\hline Gignac & $1.09 \mathrm{~m}$ & $1.46 \mathrm{~m}$ & $1.68 \mathrm{~m}$ \\
\hline
\end{tabular}

\section{B. The channel of Gignac}

In this paper, we have also studied a channel which is located in Gignac (France). The following set of parameters of this channel is considered: $L=2272 \mathrm{~m}$ is the length of the channel, $b=3 \mathrm{~m}$ is the width of the channel, $N=40$ is the number of the discretizated points, $Z_{L}$ is the water height to regulate such that $1.7 \mathrm{~m}<Z<2.5 \mathrm{~m}$.

Figure (2.a) shows that the output converges to the reference over a wide operating range. In Fig. (2.b), the water flows at the upstream and downstream stay in physical proportion, which is an important practical point.

\section{CONCLUSION}

First attempts of a Multi-Models approach on irrigation channels control, through an IMBC structure, have been realized some years ago [11]. Good experimental results were obtained 


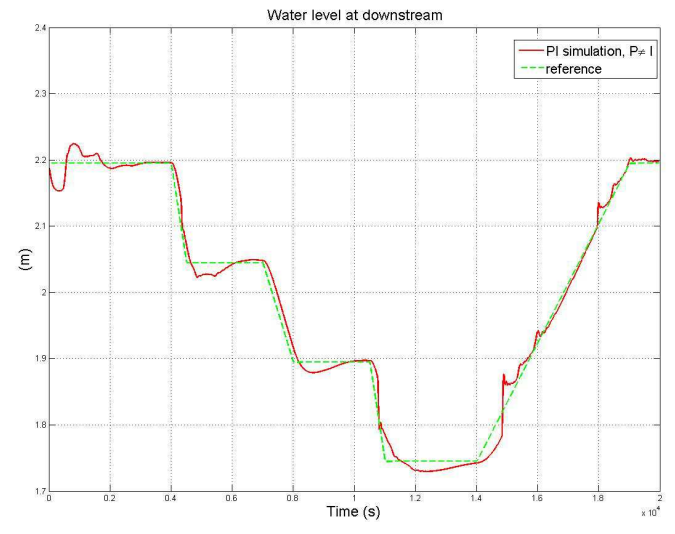

a) Comparison of the downstream water level
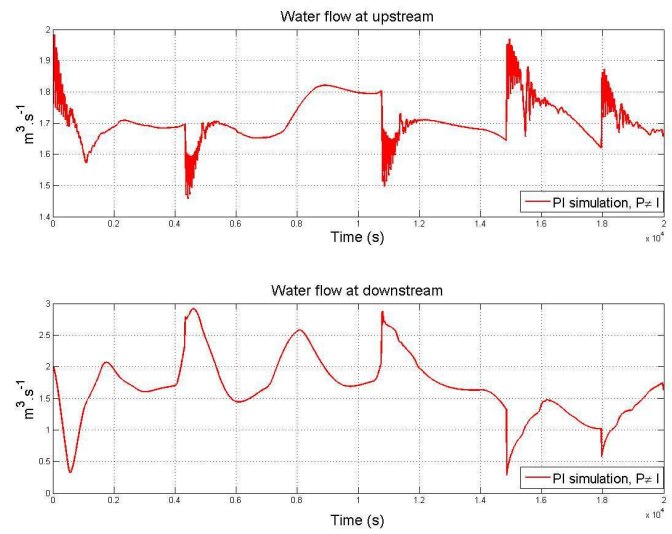

b) Water flows

Fig. 2. Gignac channel simulation

which showed promising results but a theoretical basis was lacking. The first theoretical results in order to design the feedback gain through LMI have been realized in the case of an Integral controller in [13]. Preliminary results of a PI controller in a particular case $\left(K_{i n t}=K_{p r}\right)$ have been published in [12] for infinite dimensional systems and with $K_{\text {int }} \neq K_{p r}$ in [29] for finite dimensional systems. In the present paper, the authors take into account the more general case of PI controller with $K_{i n t} \neq K_{p r}$ for infinite dimensional systems. They synthesize the new PI controller feedback gains by solving a BOI problem. Simulations show a better performance than the previous results, through this new PI feedback controller designed by BMI \& LMI [13]. The forthcoming experimentation should emphasize the improvements of the new PI controller with weighting functions, non-piecewise constant $\left(\mu_{i}(\zeta(t)) \in[0,1]\right)$.

\section{APPENDIX A}

\section{Proof OF THE PROPOSITION 1}

Proposition 4: Let $Z$ be a Hilbert space and let $G, U, V, X$ four linear operators on $Z$ such that $G: D(G) \subset Z \rightarrow D(G) . U, V$ and $X$ are densely defined on $D(G)$, the domain of $G . X$ is a self-adjoint operator such that $\|X\| \leq 2 \sigma$. If $\exists \sigma \in \mathbb{R}$ which satisfy, $\forall \phi, \varphi \in D(G)$

$$
\begin{aligned}
& \left\langle G \varphi+U^{*} \phi, \varphi\right\rangle+\left\langle U \varphi+\sigma^{-1} \phi, \phi\right\rangle<0, \\
& \left\langle G \varphi+V^{*} \phi, \varphi\right\rangle+\left\langle V \varphi+\sigma^{-1} \phi, \phi\right\rangle<0,
\end{aligned}
$$


then, the following inequality is also satisfied:

$$
\langle G \varphi, \varphi\rangle+\left\langle U^{*} X V \varphi, \varphi\right\rangle+\left\langle V^{*} X U \varphi, \varphi\right\rangle<0
$$

Proof: The objective is to prove this equation (44):

$$
\begin{array}{r}
\langle G \varphi, \varphi\rangle+\left\langle U^{*} X V \varphi, \varphi\right\rangle+\left\langle V^{*} X U \varphi, \varphi\right\rangle<0 \\
\Rightarrow\langle G \varphi, \varphi\rangle+\langle X V \varphi, U \varphi\rangle+\langle X U \varphi, V \varphi\rangle<0 \\
\langle G \varphi, \varphi\rangle+\langle V \varphi, X U \varphi\rangle+\langle U \varphi, X V \varphi\rangle<0
\end{array}
$$

Developing (43), one can obtain the following inequalities:

$$
\begin{gathered}
\left\{\begin{array}{l}
\langle G \varphi, \varphi\rangle+\left\langle U^{*} \phi, \varphi\right\rangle+\langle U \varphi, \phi\rangle+\sigma^{-1}\|\phi\|^{2}<0, \\
\langle G \varphi, \varphi\rangle+\left\langle V^{*} \phi, \varphi\right\rangle+\langle V \varphi, \phi\rangle+\sigma^{-1}\|\phi\|^{2}<0 .
\end{array}\right. \\
\Leftrightarrow\left\{\begin{array}{l}
\langle G \varphi, \varphi\rangle+\langle\phi, U \varphi\rangle+\langle U \varphi, \phi\rangle+\sigma^{-1}\|\phi\|^{2}<0 \\
\langle G \varphi, \varphi\rangle+\langle\phi, V \varphi\rangle+\langle V \varphi, \phi\rangle+\sigma^{-1}\|\phi\|^{2}<0
\end{array}\right.
\end{gathered}
$$

Let consider firstly that $\sigma$ is positive, $\phi=X V \varphi$ and $\phi=X U \varphi$ in equations (47), one gets:

$$
\left\{\begin{array}{l}
\langle G \varphi, \varphi\rangle+\langle X V \varphi, U \varphi\rangle+\langle U \varphi, X V \varphi\rangle+\sigma^{-1}\|X V \varphi\|^{2}<0, \\
\langle G \varphi, \varphi\rangle+\langle X U \varphi, V \varphi\rangle+\langle V \varphi, X U \varphi\rangle+\sigma^{-1}\|X U \varphi\|^{2}<0
\end{array}\right.
$$

Summing those both inequalities of (48), one obtains:

$$
2\{\langle G \varphi, \varphi\rangle+\langle X V \phi, U \varphi\rangle+\langle U \varphi, X V \phi\rangle\}+\sigma^{-1}\left(\|X V \varphi\|^{2}+\|X U \varphi\|^{2}\right)<0
$$

With $\sigma^{-1}>0$, it is equivalent to:

$$
\Leftrightarrow\langle G \varphi, \varphi\rangle+\langle V \varphi, X U \varphi\rangle+\langle U \varphi, X V \varphi\rangle<0
$$

So the inequality (46) and also the inequality (44) have been proved, the proposition is verified. Let consider now that $\sigma^{-\mathbf{1}}<\mathbf{0}$, four constants $\alpha, \beta, \gamma, \delta \in \mathbb{R}$, and the inequalities (47), with this time $\phi=\alpha U \varphi+\beta X V \varphi$ and $\phi=\gamma V \varphi+\delta X U \varphi$. One can obtained the following 
inequalities:

$$
\begin{array}{r}
\left\{\begin{array}{l}
\langle G \varphi, \varphi\rangle+\langle\phi, U \varphi\rangle+\langle U \varphi, \phi\rangle+\sigma^{-1}\|\phi\|^{2}<0, \\
\langle G \varphi, \varphi\rangle+\langle\phi, V \varphi\rangle+\langle V \varphi, \phi\rangle+\sigma^{-1}\|\phi\|^{2}<0
\end{array}\right. \\
\Rightarrow\left\{\begin{array}{r}
\langle G \varphi, \varphi\rangle+\langle\beta X V \varphi, U \varphi\rangle+\langle U \varphi, \beta X V \varphi\rangle \\
+\langle\alpha U \varphi, U \varphi\rangle+\langle U \varphi, \alpha U \varphi\rangle+\sigma^{-1}\|\alpha U \varphi+\beta X V \varphi\|^{2}<0, \\
\langle G \varphi, \varphi\rangle+\langle\delta X U \varphi, V \varphi\rangle+\langle V \varphi, \delta X U \varphi\rangle \\
+\langle\gamma V \varphi, V \varphi\rangle+\langle V \varphi, \gamma V \varphi\rangle+\sigma^{-1}\|\gamma V \varphi+\delta X U \varphi\|^{2}<0 .
\end{array}\right.
\end{array}
$$

Let sum both equations, then one finds:

$$
\begin{array}{ll}
\Rightarrow \quad 2\langle G \varphi, \varphi\rangle+\left(\beta+\delta+\sigma^{-1}(\alpha \beta+\gamma \delta)\right)[\langle X V \varphi, U \varphi\rangle+\langle U \varphi, X V \varphi\rangle]+\sigma^{-1} \beta^{2}\langle X V \varphi, X V \varphi\rangle \\
& +\left(2 \alpha+\sigma^{-1} \alpha^{2}\right)\langle U \varphi, U \varphi\rangle+\left(2 \gamma+\sigma^{-1} \gamma^{2}\right)\langle V \varphi, V \varphi\rangle+\sigma^{-1} \delta^{2}\langle X U \varphi, X U \varphi\rangle \\
\leq \quad & 2\langle G \varphi, \varphi\rangle+\left(\beta+\delta+\sigma^{-1}(\alpha \beta+\gamma \delta)\right)[\langle X V \varphi, U \varphi\rangle+\langle U \varphi, X V \varphi\rangle] \\
+ & \left(2 \alpha+\sigma^{-1}\left[\alpha^{2}+\|X\|^{2} \delta^{2}\right]\right)\langle U \varphi, U \varphi\rangle+\left(2 \gamma+\sigma^{-1}\left[\gamma^{2}+\|X\|^{2} \beta^{2}\right]\right)\langle V \varphi, V \varphi\rangle<0 .
\end{array}
$$

The aim is to defined the constants $\alpha, \beta, \gamma, \delta \in \mathbb{R}$ such that $\{(52) \Rightarrow(44)\}$ is satisfied $\forall$ $\sigma^{-1}<0$. Three constraints appear:

1) $\beta+\delta+\sigma^{-1}(\alpha \beta+\gamma \delta)=2$,

2) $\left(2 \alpha+\sigma^{-1}\left[\alpha^{2}+\delta^{2}\|X\|^{2}\right]\right)>0$

3) $\left(2 \gamma+\sigma^{-1}\left[\gamma^{2}+\beta^{2}\|X\|^{2}\right]\right)>0$.

If they are satisfied then $\langle G \varphi, \varphi\rangle+\langle X V \varphi, U \varphi\rangle+\langle U \varphi, X V \varphi\rangle<0$.

Consider the first constraint and let define $\alpha=\gamma=-\sigma m$, where $m \in \mathbb{R}$, then the first equality implies that $\beta+\delta=\frac{2}{1-m} m \neq 1$.

As $\beta$ and $\delta$ have the same role, we define for $m \neq 1 \beta=\delta=\frac{1}{1-m}$ then

$$
\begin{aligned}
\left(2 \alpha+\sigma^{-1}\left[\alpha^{2}+\delta^{2}\|X\|^{2}\right]\right) & =-2 \sigma * m+\sigma m^{2}+\frac{\sigma^{-1}\|X\|^{2}}{(1-m)^{2}}, \\
\left(2 \alpha+\sigma^{-1}\left[\alpha^{2}+\delta^{2}\|X\|^{2}\right]\right)>0 & \Leftrightarrow \sigma^{2} m(2-m)(1-m)^{2}>\|X\|^{2} \text { iff } 0<m<2
\end{aligned}
$$

Idem for the third inequality, as the coefficients are equals.

So, for every $\sigma<0, \exists 0<m<2, m \in \mathbb{R} \backslash\{1\}$ and an operator $X$ self-adjoint such that

$$
\sigma \sqrt{m(2-m)}(1-m)<-\|X\|
$$


and the three constraints above are satisfied with $\alpha=\gamma=-\sigma m$ and $\beta=\delta=\frac{1}{1-m}$. As it is true for all $m$, let take $m=1-\sqrt{2} / 2$, then $\sqrt{m(2-m)}(1-m)=0.5$ and one gets $\sigma<-2\|X\|$. Then, the inequality (52) is realized and (46) $\Leftrightarrow(44)$ is too. The proposition is proved.

Remark 5: The choice of $m$ is arbitrary, and one can consider another one $(m \neq 1,0<$ $m<2$ ). Here we have chosen the critical case i.e. the minimum value of the inverse function of $m(2-m)(1-m)^{2}$.

\section{REFERENCES}

[1] Alizadeh Moghadam A, Aksikas I, Dubljevic S, Forbes J. 2011. LQR control of an infinite dimensional time-varying cstr-pfr system, 18th IFAC World Congress, August 28-September 2, Milano, Italy.

[2] Saurabh Amin, Falk M. Hante, Alexandre M. Bayen. 2012. Exponential Stability of Switched Linear Hyperbolic InitialBoundary Value Problems. IEEE Transactions on Automatic Control, Vol. 57, N 2, February, 291-301.

[3] Bhagwat A, Srinivasan R, Krishnaswamy P R. 2003. Multi-linear model-based fault detection during process transitions. Chemical Engineering Science 58, 1649-1670.

[4] Boyd S, El Ghaoui L, Feron E, Balakrishnan V. 1994. Linear Matrix Inequalities in System and Control Theory, Society for Industrial and Applied Mathematics, Philadelphia, USA.

[5] Coron J M, d'Andréa Novel B, Bastin G. 2007. A strict Lyapunov function for boundary control of hyperbolic systems of conservation laws. IEEE Transactions on Automatic Control 52(1), 2-11.

[6] Curtain R F, Zwart H . 1995. An introduction to Infinite Dimensional Linear Systems, Springer Verlag, New York.

[7] Dashkovskiy, S. and Mironchenko, A., Input-to-state stability of infinite-dimensional control systems, Mathematics of Control, Signals, and Systems March 2013, Volume 25, Issue 1, pp 1-35.

[8] de Saint-Venant A B. 1871. Théorie du mouvement non permanent des eaux avec applications aux crues des rivières et à l'introduction des marées dans leur lit. Comptes rendus de l'Académie des Sciences de Paris 73, 148-154, 237-240.

[9] Dos Santos V, Bastin G, Coron J M, d'Andréa Novel B. 2008. Boundary control with integral action for hyperbolic systems of conservation laws: Lyapunov stability analysis and experimental validation. Automatica 44(5), 1310 - 1318.

[10] Dos Santos V, Prieur C. 2008. Boundary control of open channels with numerical and experimental validations. IEEE Transactions on Control Systems Technology 16, 1252-1264.

[11] Dos Santos V, Toure Y, Mendes E, Courtial E. 2005. Multivariable Boundary Control approach by internal model, applied to irrigations canals regulation. In: Proc. 16th IFAC World Congress, Prague, Czech Republic.

[12] Dos Santos Martins V, Rodrigues M. 2011. A Proportional Integral Feedback for Open Channels Control Trough LMI Design, 18th IFAC World Congress, August 28 - September 2, 2011, Milano, Italy.

[13] Dos Santos Martins V, Rodrigues M, Diagne M. 2012. A Multi-Models Approach of Saint-Venant's Equations: A Stability study LMI. Int. Jour. of Applied Mathematics and Computer Science, Vol.22, No.3, September,2012

[14] Dulhoste J F, Besançon G, Georges D. 2001. Nonlinear control of water flow dynamics by input-output linearisation based on a collocation model. European control conf., Porto, Portugal.

[15] H.O. Fattorini, Boundary Control Systems, SIAM J. Control, 6, 3, 1968.

[16] Gatzke E, Doyle F. 2002. Use of multiple models and qualitative knowledge for on-line moving horizon disturbance estimation and fault diagnosis. Journal of Process Control 12, 339-352. 
[17] Georges D, Litrico X. 2002. Automatique pour la Gestion des Ressources en Eau. Edts IC2, Systèmes automatisés, Hermès.

[18] Greenberg J M, Li T. 1984. The effect of boundary damping for the quasilinear wave equations. Journal of Differential Equations 52, 66-75.

[19] Leith D J, Leithead W E. 2000. Survey of gain-scheduling analysis and design. International Journal of Control 73 (11), $1001-1025$.

[20] Li T. 1994. Global Classical Solutions for Quasilinear Hyperbolic Systems. Research in Applied Mathematics. Masson and Wiley, Paris, Milan, Barcelona.

[21] Litrico X, Fromion V. 2006. $H_{\infty}$ control of an irrigation canal pool with a mixed control politics. IEEE Trans. on Control Systems Technology 14(1), 99-101.

[22] Litrico X, Georges D. 1999a. Robust continuous-time and discrete-time flow control of a dam-river system: (i) modelling \& (ii) controller design. J. of Applied Mathematical Modelling 23(11), 809-827 \& 829-846.

[23] Malaterre P O, Rogers D, Schuurmans J. 1998. Classification of canal control algorithms. J. of Irrigation and Drainage Engineering 124(1), 3-10.

[24] Mareels I, Weyer E, Ooi S, Cantoni M, Li Y, Nair G. 2005. Systems engineering for irrigation systems: Successes and challenges. Annual Reviews in Control 29(2), 191-204.

[25] Mazenc, F., Prieur, C., Strict Lyapunov functions for semilinear parabolic partial differential equations, Mathematical Control and Related Fields 1, 231-250 (2011)

[26] Murray-Smith R, Johansen T. 1997. Multiple Model Approaches to Modelling and Control. Taylor and Francis.

[27] Papageorgiou M, Messmer A. 1989. Flow control of a long river stretch. Automatica 25(2), 177-183.

[28] Rodrigues M., Sahnoun M., Theilliol D., Ponsart J.-C. 2013. Sensor Fault Detection and Isolation Filter for Polytopic LPV Systems: A Winding Machine Application. Journal of Process Control 23, Issue 6 (2013) 805-816.

[29] Rodrigues M., Wu Y., Aberkane S. and Dos Santos Martins, V.. 2013. LMI \& BMI Technics for the Design of a PI Control for Irrigation Channels, ECC 2013, Zurich, July 17-19, 2013.

[30] Rodrigues M, Theilliol D, Aberkane S, Sauter D. 2007. Fault tolerant control design for polytopic LPV systems. Int. Journal. Applied Math. Comput. Sciences 17 (1), 27-37.

[31] A. Sasane and R.F. Curtain. 2001. Optimum Hankel Norm Approximation for the Pritchard-Salamon class of infinitedimensional systems, J. Integral Equations and Operator Theory, 39, 2001, 98-126.

[32] Skelton R, Iwasak T, Grigoriadis K. 1997. A Unified Algebraic Approach to Linear Control Design. Taylor and Francis, London, UK.

[33] Touré Y, Rudolph J. 2002. Controller design for distributed parameter systems, Encyclopedia of LIFE Support on Control Systems, Robotics and Automation I:933-979.

[34] Triggiani R. 1975. On the stability problem in banach space, Journal of Math. Anal. and Appl. 52: $383-403$.

[35] Weyer E. 2002. Decentralised PI controller of an open water channel. 15th IFAC world congress, Barcelona, Spain.

[36] Zaccarian L, Li Y, Weyer E, Cantoni M, Teel A R. 2007. Anti-windup for marginally stable plants and its application to open water channel control systems. Control Engineering Practice 15(2), 261-272. 